\title{
Applying a Chiral Mechanical "Field" to an Achiral Liquid Crystal
}

\section{Macroscopic Torsional Strain and Induced Molecular Conformational Deracemization}

R. Basu, J. S. Pendery, R. G. Petschek, R. P. Lemieux, and C. Rosenblatt

Physical Review Letters 107, 237804 (2011), published 2 December 2011

DOI:10.1103/PhysRevLett.107.237804

\section{Recommended with a commentary by Jonathan V. Selinger, Kent State University}

Throughout the physical and biological world, many objects and materials are clearly different from their mirror images. A classic example is the human hand: a right hand is not equivalent to a left hand, and it cannot be superimposed on a left hand by any combination of rotations or translations. This asymmetry between an object and its mirror image is called chirality. Chirality is common in organic chemistry; it usually occurs if an organic molecule includes a carbon atom that is tetrahedrally bonded to four inequivalent groups. Indeed, living organisms are always chiral, as can be seen by the double-helical structure of DNA. As a result, the interaction of a right-handed molecule with the human body is different from the interaction of the mirror-image left-handed molecule with the body. This distinction is very important for pharmaceuticals, because one handedness of a molecule may be beneficial while the mirror image is inert or even harmful.

Chirality is also important for liquid-crystal science and technology, because it changes the orientational order of molecules. If liquid-crystal molecules are not chiral, they tend to align parallel to their neighbors, forming a nematic phase with long-range orientational order. By contrast, if liquid-crystal molecules are chiral, the chiral asymmetry favors a helical twist in the molecular orientation, called the director, leading to cholesteric or chiral nematic phase. This twist changes the interaction of the phase with applied fields and with polarized light, and can be an essential part of liquid-crystal applications.

Organic chemists tend to view chirality as a fixed, static property of molecules. Indeed, they work hard to synthesize extremely pure right- or left-handed compounds for drugs, liquid crystals, or other applications. By contrast, for theoretical physicists with a background in statistical mechanics, it is natural to think of chirality as a symmetry-breaking order parameter, analogous to the net magnetization in the classic Ising model of magnetism. In this analogy, the chiral order parameter is a positive or negative number that describes which way the reflection symmetry is broken (into a right- or left-handed state), and how much it is broken. ${ }^{1}$ In some experiments, the chiral order parameter might be saturated near the maximum value of \pm 1 , if molecules are stuck in some chiral conformation and never leave it, as in a pure chiral drug. In other experiments, the chiral order parameter might fluctuate around 0 , if molecules are moving in and out of chiral conformations separated by small energy barriers.

If we think of chirality as an order parameter that can change during an experiment, then we would expect to see a phase transition in which the reflection symmetry is spontaneously broken. At temperatures above this transition, the chiral order parameter is zero and the system is achiral, but below this transition the order parameter becomes nonzero and the system becomes chiral. If the system is a liquid crystal, then the chiral order parameter must be coupled to twist in the molecular director, leading to a local cholesteric structure. Several years ago, my collaborators and I worked on a theory for chiral ordering coupled to director distortions in Langmuir monolayers and liquid-crystal films, and found that the system forms alternating stripes of right- 
handed chirality with one sign of director rotation, followed by left-handed chirality with the opposite sign of director rotation. ${ }^{2}$ The best experimental realization of this effect has probably been work from the Colorado group, which shows a transition from a uniform achiral state to a state with alternating stripes. ${ }^{3}$

Based on this view of chirality as an order parameter, we should also expect the disordered achiral phase to respond linearly to a symmetry-breaking field. The field could be any external influence that breaks the symmetry between the system and its mirror image-for example, applying parallel electric and magnetic fields, or doping the system with chiral impurities. Such an influence would eliminate the degeneracy between right- and left-handed states, e.g., between right- and left-handed chiral conformations of the molecules. As a result, it would induce at least a slight population difference between these states, and the macroscopic properties of the system would become chiral. This response to symmetry-breaking fields has been seen in a class of helical polymers called polyisocyanates, in which cooperativity along the polymer chains leads to a strong amplification of the chiral effects. ${ }^{4}$ However, it is generally not observable in liquid crystals, just because it is a very small effect.

The current article by researchers at Case Western Reserve University and Queen's University describes a new approach to measure the chiral order parameter in response to a symmetry-breaking field using liquid crystals. ${ }^{5}$ Here, the authors create a chiral mechanical "field" through the coupling between chirality and director twist in liquid crystals. By rubbing the substrates, they establish easy axes on the two sides of a liquid-crystal cell. As shown in Fig. 1, these easy axes are an angle $\theta_{0} \approx 20^{\circ}$ apart from each other, and hence the liquid-crystal director must twist through $20^{\circ}$ across the thickness of the cell. This twist couples to the chiral order of the liquid-crystal molecules, breaking the symmetry between right- and left-handed molecular

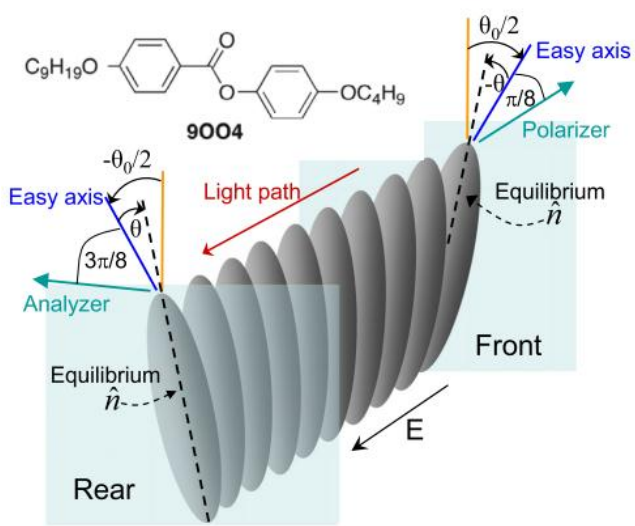

Fig. 1. Schematic diagram of experimental geometry for twist cell, from Ref. 5. conformations. As a result, it induces a population difference between these conformations, known as deracemization. By using a wedge-shaped cell, with a thickness varying from zero to $12.5 \mu \mathrm{m}$, the experiment varied the magnitude of the twist and hence the chiral symmetry-breaking field, and probed the magnitude of deracemization as a function of applied twist.

The symmetry breaking is still very small. Through a model involving elastic energy vs. deracemization entropy, the authors estimate that the relative population difference between right- and left-handed conformers at the center of the cell is of order $10^{-5}$, which is too small to observe. To get a detectable population difference, they take advantage of the surface electroclinic effect, which is an extra twist over a narrow region of a few nanometers near the surface of any chiral liquid crystal under an applied electric field. Here, the induced chirality is sufficient to generate a surface electroclinic effect. The extra surface twist associated with this effect amplifies the chiral population difference near the surface to $10^{-3}$, and this surface population difference is large enough to observe. The measurement is done through electro-optic techniques, which involve analyzing and modeling the surface electroclinic effect itself.

One possible extension of this research would be to modify the setup to make the symmetrybreaking effect easier to see. For example, one might scribe alternating domains of different 
easy axes on the cell surfaces in order to create lateral domains of right- and left-handed twist inside the liquid crystal. This twist would establish a mechanical chiral field that alternates in sign as a function of lateral position across the cell. If the liquid crystal is achiral, as in the current experiment, then the population of right- and left-handed chiral conformers would also vary as a function of position. By comparison, if the liquid crystal were a mixture of noninterconverting right- and left-handed molecules, then it should actually phase separate into domains of alternating composition. The two chemical components would not even have to be mirror images of each other; they could just be two liquid-crystal compounds with different favored twists. The induced phase separation of these components might be easier to see than the surface electroclinic effect that is measured here.

An even more intriguing possibility would be to work with liquid crystals that have a chiral symmetry-breaking transition, like the materials in Ref. 3. If this transition is second-order, then the susceptibility to a chiral field should diverge at the transition temperature. In other words, if the liquid crystals are on the verge of spontaneous chiral order, they should become extremely sensitive to any slight chiral influences, such as the mechanical twist in Ref. 5. Hence, the population difference between chiral conformers should become very large and temperaturedependent just above this transition. Direct observation of this effect would further demonstrate the concept of chirality as an order parameter that can be manipulated by external fields.

I would like to thank Robin Selinger for helpful discussions about this work.

${ }^{1}$ For a review of chiral order parameters, including some surprising subtleties in the definition of these parameters, see A. B. Harris, R. D. Kamien, and T. C. Lubensky, Reviews of Modern Physics 71, 1745 (1999).

2 J. V. Selinger, Z.-G. Wang, R. F. Bruinsma, and C. M. Knobler, Physical Review Letters 70, 1139 (1993).

${ }^{3}$ J. Pang and N. A. Clark, Physical Review Letters 73, 2332 (1994).

${ }^{4}$ M. M. Green, J.-W. Park, T. Sato, A. Teramoto, S. Lifson, R. L. B. Selinger, and J. V. Selinger, Angewandte Chemie International Edition 38, 3138 (1999).

${ }^{5}$ R. Basu, J. S. Pendery, R. G. Petschek, R. P. Lemieux, and C. Rosenblatt, Physical Review Letters 107, 237804 (2011). 